STONE DISEASE

\title{
Renal functional effects of multiple-tract percutaneous access
}

Handa RK, Evan AP, Willis LR, Johnson CD, Connors BA, Gao S, Lingeman JE, Matlaga BR, Miller NL, Handa SE

Department of Anatomy and Cell Biology, Indiana University School of Medicine, Indianapolis, Indiana, USA

J Endourol. 2009; 23: 1951-6

Introduction: Percutaneous nephrolithotomy (PCNL) can involve establishing more than one access into the urinary collecting system. The present study examined whether multiple percutaneous accesses results in a more severe reduction in renal function than that after single-percutaneous access.

Methods: Adult female pigs were anesthetized, and percutaneous access to the left urinary collecting system was achieved by puncturing the lower pole calyx (single-tract access, $n=16$ ) or serially puncturing the lower pole, interpolar region, and upper pole calyces [multiple (three)-tract access, $n=11$ ]. Renal function measurements included glomerular filtration rate and effective renal plasma flow, and were taken immediately before and 1.5 and 4.5 hours after percutaneous access. We also examined glomerular function in a group of adult patients with normal preoperative serum creatinine $(\mathrm{Cr})$ levels $(<\mathrm{or}=1.4 \mathrm{mg} / \mathrm{dL})$ who underwent either unilateral single-tract PCNL (23 patients) or unilateral multiple (two)-tract PCNL (10 patients). Access tracts were dilated to 30F with a NephroMax balloon dilator system in animal and human patients.

Results: Single- and multiple-tract percutaneous access procedures in pigs resulted in a similar renal functional response; both glomerular filtration rate and effective renal plasma flow significantly declined by approximately $60 \%$ immediately after access and remained depressed throughout the experimental observation period. A retrospective analysis of patients with normal serum $\mathrm{Crs}(<\mathrm{or}=1.4 \mathrm{mg} / \mathrm{dL})$ who underwent single- or multiple-tract PCNL demonstrated that the procedures produced similar and significant increases in serum $\mathrm{Cr}$ on postoperative day $1(0.33+/-0.09$ [standard error of mean] $\mathrm{mg} / \mathrm{dL}$ and $0.39+/-0.11 \mathrm{mg} / \mathrm{dL}$, respectively) and day $2(0.33+/-0.09 \mathrm{mg} / \mathrm{dL}$ and $0.25+/-0.09 \mathrm{mg} / \mathrm{dL}$, respectively).

Conclusions: Multiple-tract access does not lead to a more severe reduction in renal function than single-tract access; that is, the acute renal hemodynamic response to PCNL appears independent of the number of access tracts.

\section{Editorial Comment}

The human study is limited as there were significant differences in baseline renal function between the two groups analyzes. Creatinine clearance calculations based on spot serum levels is a relatively crude measure of renal function, and could also be impacted by anemia, hydration and medications in the perioperative period.

In the porcine study, though changes in ipsilateral kidney function were marked ( $>60 \%$ decrease GFR and RPF), no difference was noted whether one or three tracts were created. In contrast, multiple tract access appeared to have a greater impact on the contralateral untreated kidney, with greater decreases in GFR and RPF $(>45 \%$ vs. $<20 \%)$. Though this did not reach statistical significance, it does warrant concern - suggesting that greater caution is warranted at least in the perioperative period if multiple-tract access is utilized with regards to using medications that rely on renal clearance or have the potential for nephrotoxicity. Long-term prospective studies evaluating the relative impact of multiple vs. single tract access with more liberal use of flexible nephroscopy and/or ureteroscopy as an adjunct are warranted. 